\title{
LA INSTRUCCIÓN Y LA ENSEÑANZA EN LA PROVINCIA DE ALICANTE DURANTE EL SIGLO XX
}

\author{
Pascual-Antonio Bartolomé Pina
}

\begin{abstract}
RESUMEN
Este artículo analiza la evolución que han experimentado la enseñanza y la instrucción de los habitantes de la provincia de Alicante en el último siglo. De este análisis se desprenden, entre otras, las siguientes conclusiones: por un lado, que existe una estrecha relación entre la estructura educativa de un país y su estructura socioeconómica, y por otro, que la enseñanza y la instrucción de los habitantes de un determinado territorio suele atravesar tres etapas en su desarrollo, que en nuestro caso son la preinstructiva (hasta 1940), la de la instrucción elemental (hasta 1970) y etapa de las reformas educativas, en que nos encontramos actualmente.
\end{abstract}

\begin{abstract}
This article analyses the evolution experienced by the education and the training of the population from the Province of Alicante in the last century. The following conclusions are implied by this analysis: On one hand, that there is a close relation ship between the educative structure of a country and its socioeconomic structure, and on the other, that the education and training of the population of a specific territory usually goes through three stages in its development, which, in our case is the preinstructive (up to 1940), elemental instruction (up to 1970) and the period of educational changes, where we are nowadays.
\end{abstract}

\section{Introducción}

La evolución que ha experimentado durante el presente siglo la enseñanza que reciben y la instrucción que poseen nuestros ciudadanos, ha permitido que éstas hayan dejado de ser un «derecho de los burgueses» ${ }^{1}$ para convertirse «...en una función fundamental ejercida, si bien en diferente medida, por todas las capas de la población...»². Precisamente esta

\footnotetext{
${ }^{1}$ Véase la obra de MAIER, J.; PAESLER, R.; RUPPERT, K.; SCHAFFER, F.: Geografía Social, RIALP, Madrid, 1987, p.109.

${ }^{2}$ Véase MAIER, J. y otros: op. cit., p. 110.
} 
forma diferente de recibir la enseñanza, según se pertenezca a un estrato social u otro o según sea el tipo de entidad espacial en donde se viva, la convierte en un importante objeto de estudio de la Geografía.

La escasa consideración que en los trabajos geográficos ha tenido hasta fechas recientes el comportamiento de la población ante la enseñanza ${ }^{3}$, se debe quizás a que el ejercicio de esta función crea instalaciones que conforman el paisaje de una manera menos amplia que las funciones «vivienda» o «trabajo», por citar sólo algunas de las más estudiadas. Sin embargo, hoy en día son cada vez más numerosos los geógrafos ${ }^{4}$ que llaman la atención sobre la importancia que tienen la enseñanza y la instrucción como fuerzas conformadoras del paisaje cultural, tanto desde el punto de vista espacial como desde la pragmática óptica del planificador territorial, pues la educación que reciben o poseen los ciudadanos de un determinado territorio, puede ser un perfecto indicador sociocultural, económico e incluso político del mismo 5 .

Las principales fuentes que se han consultado en la elaboración de este trabajo han sido los Censos de población de los años comprendidos entre 1887 y 1981, los Padrones Municipales de habitantes de 1975 y 1986, así como diversas publicaciones del Instituto Nacional de Estadística, de la Consellería de Cultura, Educació i Ciència de la Generalitat Valenciana y del Ministerio de Educación y Ciencia.

\section{El analfabetismo en la provincia de Alicante}

\subsection{Introducción}

El término analfabeto es simplemente lo contrario de alfabeto, y éste es aquél que sabe leer y escribir. Sin embargo, al observar la realidad, nos encontramos con varias particularidades; así, hay quien sabe leer pero no escribir; igualmente, hay mucha gente que sabe leer y escribir, pero difícilmente entiende lo que lee y a duras penas sabe escribir algo más que su firma -son los que cabe llamar semianalfabetos-; todo ello sin citar a aquellos que teniendo la capacidad para usar la palabra escrita, no la explotan, bien porque no la necesitan o bien porque no se sienten motivados a hacerlo -éstos podrían ser denominados analfabetos prácticos-.

Por todo ello y sin olvidar la enorme importancia que tienen estos matices según sea el nivel instructivo general de la población, empezaremos por definir las dos principales categorías de analfabetos que podemos encontrar; así, entendemos por analfabeto absoluto o simplemente analfabeto, a aquella persona que, con 10 o más años de edad, no sabe leer ni escribir, y como analfabeto funcional a la que, si bien teóricamente sabe leer y escribir, es incapaz de comprender la lectura de un texto de nivel periodístico o de exponer por escrito hechos de su vida cotidiana.

\footnotetext{
${ }^{3}$ En los últimos años se han publicado trabajos muy interesantes relacionados con el tema, de entre ellos no podemos dejar de señalar uno que fue casi pionero en España, la Tesis Doctoral de Ana OLIVERA POLL - La enseñanza en Madrid: Análisis de una función urbana- o los estudios realizados por José Luis ANDRÉS SARASA referidos a la provincia de Murcia o por el equipo que dirige, desde la Universidad de Caen, el profesor Robert HERIN, que han sido publicados por la revista Géographie Sociale, 9, marzo de 1990.

${ }^{4}$ Véase MAIER, J. y otros: op. cit., p. 111.

${ }^{5}$ El presente artículo resume algunos aspectos de la Tesis Doctoral del autor del mismo -Geografía de la enseñanza de la provincia de Alicante-, que dirigida por el catedrático Vicente Gozávez Pérez, fue leída en el Departamento de Geografía Humana de la Universidad de alicante en 1989. En la actualidad dicha Tesis Doctoral es de publicación inminente por el Instituto de Estudios «Juan Gil Albert» dependiente de la excma. Diputación Provincial de Alicante.
} 


\subsection{Evolución de la tasa de analfabetismo}

La tasa de analfabetismo, si bien decrece de modo continuo en la provincia de Alicante entre 1887 y 1986 -cae 73,25 puntos porcentuales entre ambos años-, no lo hace en modo alguno de manera uniforme a lo largo del último siglo; así, podemos distinguir en su evolución dos periodos que, separados por el año 1950, se caracterizan: el primero por la acelerada caída de la tasa, y el segundo por el estancamiento del índice al alcanzar éste cotas bajas. En lo que respecta a la evolución que experimenta esta misma tasa en cada sexo, podemos señalar que, aunque desciende el índice tanto en los varones como en las mujeres, es mucho más importante la caída de la tasa entre la población femenina que entre la masculina -aquéllas reducen su índice en 78,81 puntos porcentuales frente a los 67,38 en que lo hacen los varones-; y ello debido, en buena medida, a que la tasa de partida femenina es más alta que la masculina -un $84,87 \%$ y un $69,43 \%$, en cada caso- (ver cuadro I y figura 1 ).

Al comparar estas cifras con las del resto del País Valenciano hemos observado que, al mismo tiempo que la tasa de analfabetismo de la provincia de Valencia es más baja que la de Alicante en cada uno de los años considerados, la de Castellón es, por el contrario, más alta, debido, en gran parte, a su menor desarrollo económico, al que contribuyó, hasta fechas recientes, su escasa industrialización; por otro lado, hemos hallado en Castellón un ritmo en el descenso del índice muy similar al de Alicante -incluso se pueden marcar los mismos dos períodos-, cosa que no ocurre en Valencia, cuya caída es mucho más irregular; finalmente, podemos señalar que las tasas de analfabetismo de las provincias de Valencia y Castellón están más influenciadas por las de sus capitales que la de Alicante, ya que al calcularlas, una vez restada la población de dichas ciudades, la tasa es algo más alta que la de Alicante (ver figura 2).

\section{Cuadro I}

TASAS DE ANALFABETISMO EN LA PROVINCIA DE ALICANTE, DESDE 1887 A 1986

Total Provincia Alicante

$\begin{array}{lcccccc}\text { Años } & \text { Varón } & \text { Mujer } & \text { Total } & \text { Varón } & \text { Mujer } & \text { Total } \\ 1887 & 69,43 & 84,87 & 77,36 & 71,58 & 86,52 & 79,22 \\ 1900 & 64,64 & 79,70 & 72,39 & 66,63 & 81,37 & 74,18 \\ 1910 & 57,74 & 72,68 & 65,51 & 60,31 & 74,79 & 67,83 \\ 1920 & 46,73 & 62,76 & 55,16 & 49,22 & 65,11 & 57,52 \\ 1930 & 34,33 & 52,05 & 43,56 & 36,28 & 54,39 & 45,68 \\ 1940 & 23,44 & 38,92 & 31,62 & 25,15 & 41,23 & 33,66 \\ 1950 & 11,84 & 22,17 & 17,24 & 24,76 & 34,52 & 29,84 \\ 1960 & 9,14 & 21,51 & 15,62 & 9,95 & 22,87 & 16,66 \\ 1970 & 5,8 & 14,93 & 10,51 & 6,4 & 15,99 & 11,31 \\ 1975 & 4,31 & 11,3 & 7,91 & 4,56 & 12,07 & 8,4 \\ 1981 & 3,65 & 10,26 & 7,05 & 3,98 & 10,97 & 7,55 \\ 1986 & 2,05 & 6,06 & 4,11 & 2,18 & 6,38 & 4,32\end{array}$

FUENTE DE LOS DATOS PRIMARIOS: Censos de población y Padrones Municipales de habitantes. Elaboración propia. 


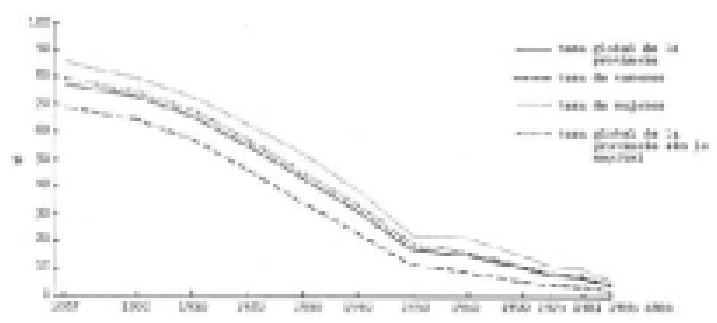

FUENTE DE LOS DATOS PRIMARIOS: Censos de 1887, 1900, 1910, 1920, 1930, 1940, 1950, 1960, 1970 y 1981 , y Padrones Municipales de habitantes de 1975 y 1986.

FIGURA 1. Evolución de las tasas de analfatebismo en la provincia de Alicante de 1887 a 1986, según el sexo.

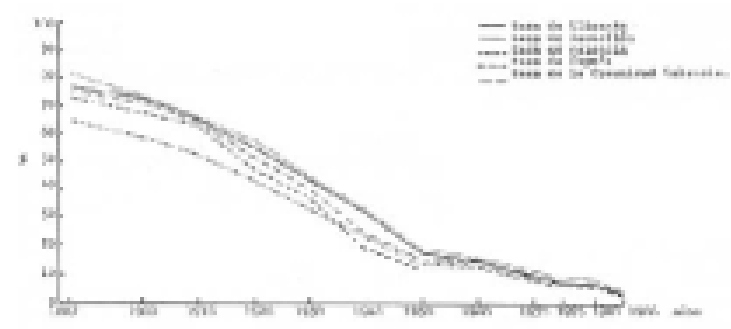

FUENTE DE LOS DATOS PRIMARIOS: Censos de 1887, 1900, 1910, 1920, 1930, 1940, 1950, 1960, 1970 y 1981, y Padrones Municipales de habitantes de 1975 y 1986.

FIGURA 2. Evolución de las tasas de analfabetismo en las provincias de Alicante, Castellón y Valencia, en la Comunidad Valenciana y en el Estado Español de 1887 a 1986, según el sexo.

\subsection{Evolución del analfabetismo por edad y sexo}

\subsubsection{Introducción}

La diferente manera con que los censos agruparon la población según su edad antes y después de 1950, así como el que la población considerada haya sido la de hecho hasta 1950 , y la de derecho desde 1960, nos ha obligado a establecer en nuestro análisis dos 
períodos. No obstante y a pesar de ello, no hemos renunciado a realizar un estudio de la evolución del analfabetismo desde 1887 hasta 1986.

\subsection{Período de 1887 a 1940}

La tasa de analfabetismo experimenta entre 1887 y 1940 la siguiente evolución en cada grupo de edad y sexo: por un lado, un descenso continuo en todos los grupos establecidos, aunque, las tasas de los varones son siempre más bajas que las de las mujeres; por otro, una caída de la tasa muy regular en cada grupo de edad y sexo -el descenso del índice sólo se desacelera entre la población de 10 a 15 años, que en los dos sexos sufre un estancamiento e incluso se incrementa entre 1900 y 1910, y entre las mujeres de más de 60 años a partir de 1900-; asimismo cabe destacar que la caída de las tasas femeninas es mayor que la de las masculinas entre los 10 y los 25 años de edad -de 4 a 6 puntos porcentuales más-, esta situación se invierte a partir de los 36 años, ya que los varones ven decrecer su índice entre 1 y 3 puntos más que las mujeres; por último, podemos señalar que mientras las tasas más bajas tienden a concentrarse entre los 16 y 35 años en los varones, y entre los 16 y 25 años en las mujeres, las más altas las hallamos siempre entre los mayores de 45 años de ambos sexos (ver figura 3 y 4 ).

Respecto a los cambios que se producen en la estructura socioeconómica de la población durante este período podemos señalar que: mientras que el número de habitantes con 10 años o más crece de 1900 a 1940 en un 41,9\%, la población activa sólo lo hace en un 36,4\%, disminuyendo la tasa de actividad dos puntos porcentuales entre ambos años; asimismo, se da una importante disminución de las personas ocupadas en el sector primario -que pasan de representar el 68,22\% del total de activos a suponer sólo el 43,56\%-, un notable incremento de los efectivos del sector secundario -que aumentan su porcentaje de un $18,11 \%$ a un $36,09 \%$-, y un claro aumento de ocupados en el sector terciario -cuya tasa pasa de representar el $13,67 \%$ a convertirse en el 20,34\% de la población activa- (ver Cuadro II).

Todo ello nos sugiere, entre otras, las siguientes conclusiones: la existencia de un importante trasvase de jóvenes del mundo laboral a los centros de enseñanza, lo que implicará, tal como veremos más adelante, un notable aumento del porcentaje de estudiantes en el total de población -este hecho afectará sobre todo a los grupos de edad más jóvenes que, salvo durante la Guerra Civil, tenderán a crecer-; por consiguiente el descenso de la tasa de analfabetismo es, en gran medida, consecuencia del aumento del porcentaje de estudiantes, más si observamos cómo aquélla disminuye al mismo ritmo en que lo hacen los índices de analfabetos en las edades más jóvenes, al mismo tiempo que se produce un cierto estancamiento de la tasa de analfabetos en los grupos con más edad -precisamente el hecho de que las mujeres, con un cierto desfase, vayan reduciendo sus tasas con un ritmo tan uniformemente acelerado como los varones lo confirma-; todo ello sin olvidar la importancia que tiene en este proceso el desarrollo de la economía provincial -su creciente industrialización con la consiguiente aparición de nuevas profesiones, obligará a muchos jóvenes a adquirir una formación mínima para poder encontrar trabajo-.

\subsubsection{Período de 1960 a 1986}

La tasa de analfabetismo, aunque sigue cayendo en cada grupo de edad y sexo entre los años 1960 y 1986, lo hace de manera cada vez menos espectacular, sobre todo entre los 

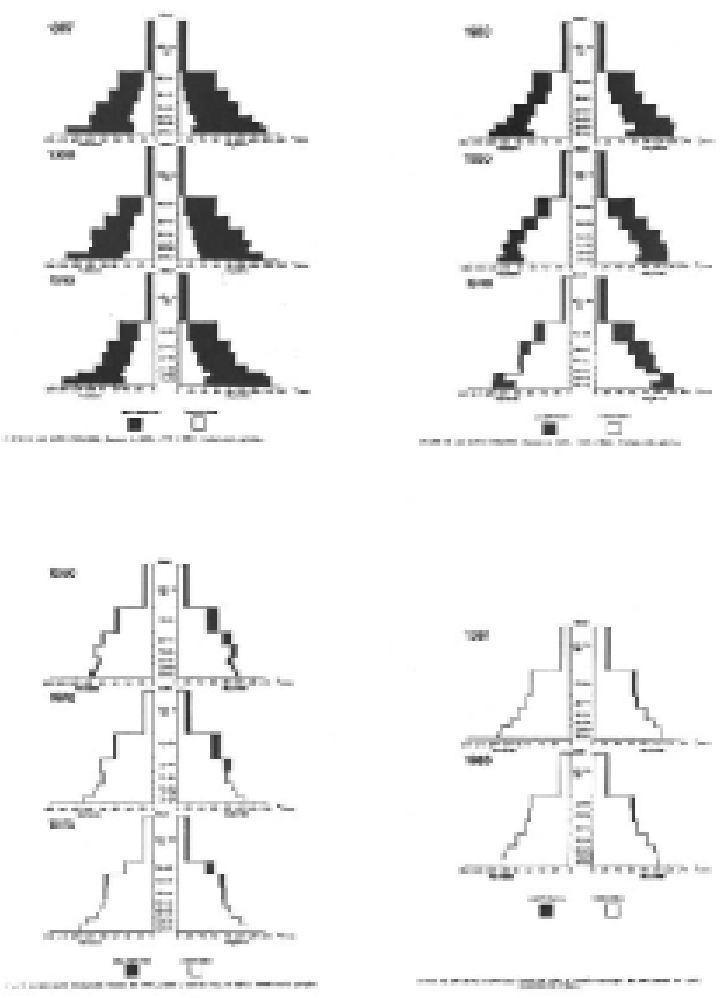

FIGURA 3. Estructura de la población de la provincia de Alicante, por edad, sexo e instrucción, en porcentajes. 


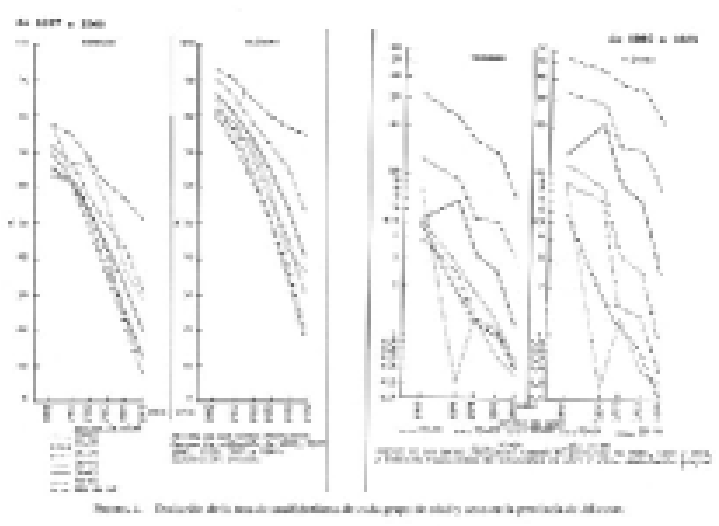

FIGURA 4. Evolución de la tasa de analfabetismo de cada grupo de edad y sexo en la provincia de Alicante. 


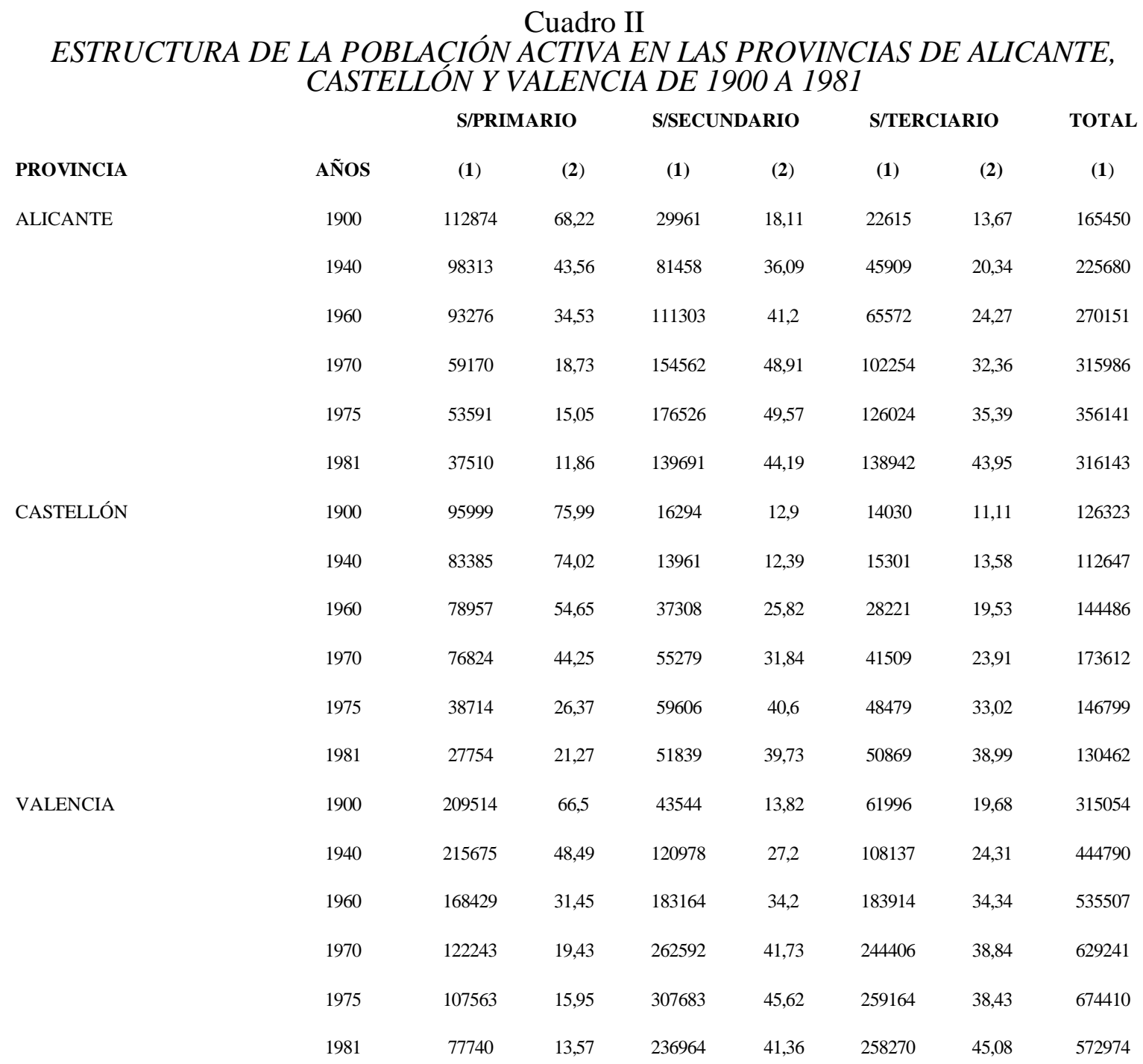

FUENTE DE LOS DATOS PRIMARIOS: Censos de población y Padrón Municipal de habitantes. Elaboración propia.

pOTA: $<$ S/> significa sector, (1) cifras absolutas y (2) \% sobre el total de cada provincia/año.

más jóvenes, llegándose a estancar el índice cuando éste desciende por debajo del 1\%. Por otra parte, en esta etapa y con respecto a la anterior, la caída de la tasa es mucho más acusada en las mujeres que en los varones, sobre todo en los grupos de población con edades más avanzadas.

Asimismo, cabe destacar que en este período se produce un progresivo acercamiento de las tasas femeninas a las masculinas, hasta acabar situándose aquéllas a un nivel similar, cuando no más bajo que el índice de los varones entre los grupos con menos de 25 años; al mismo tiempo hallamos un claro desfase entre las tasas de analfabetismo de las mujeres con más de 45 años y las de los varones con la misma edad aquéllas obtienen en 1986 porcentajes similares a los que éstos presentaban en 1970-. Por último, podemos señalar que hemos hallado un rebrote del índice de analfabetismo en los dos sexos en el grupo de 35 a 44 años de edad entre 1960 y 1970, cuya tasa, por otro lado, caerá espectacularmente entre 1970 y 1975 (ver figuras 3, 4 y 5).

Todos estos datos pueden complementarse con los que hemos obtenido de la evolución de la estructura socioeconómica de la población entre 1960 y 1986; así tenemos que mientras que la tasa de actividad apenas sube 2 puntos porcentuales entre dichos años, incrementándose el número de activos en un $62 \%$, la población con 16 años o más lo hace entre esos mismos años en un 74,2\% -se considera en este período a los habitantes con 16 años o más y no a los que tenían 10 o más años, al cambiar respecto con el anterior la edad mínima para poder entrar en el mundo del trabajo-. 
Por otro lado, entre 1960 y 1981 se produce un importante descenso de la población activa del sector primario, al caer su tasa del 34,53\% en la primera fecha al $11,86 \%$ en la segunda; en esta caída es importante el período que va de 1960 a 1970, al descender el índice de activos de dicho sector más de 14 puntos porcentuales. Asimismo, la población activa del sector secundario, si bien crece globalmente a lo largo del período casi tres puntos, no lo hace de forma uniforme -se eleva siete puntos hasta 1970, comenzando a decrecer a partir de entonces-. Por otra parte, la población activa del sector terciario no sólo eleva espectacularmente su tasa durante dichos años -pasa ésta de un $24,27 \%$ a un $43,95 \%$-, sino que además lo hace de una manera mucho más acompasada.

Asimismo, es importante señalar que entre 1960 y 1986 aumenta considerablemente la población que no ha nacido en municipios de la provincia de Alicante -pasa del 16,3\% al 26,3\% del total de población censada-. Entre éstos destaca el progresivo aumento de residentes nacidos fuera de España -su porcentaje del total de población se eleva del 0,6\% al 4,2\%-, y ello como resultado, tanto del empadronamiento en esta provincia de numerosos extranjeros, como de la llegada de muchos hijos de emigrantes que han acompañado a sus padres en su regreso definitivo a España. En cuanto a los nacidos en otras provincias, si bien a lo largo de todo el período también han crecido, el mayor salto se dio entre 1960 y 1970, momento en que suben 8 puntos porcentuales y sitúan su tasa en el 23,5\% del total de población de la provincia; sin embargo, entre 1981 y 1986 ha decrecido su peso específico, pues ha descendido su porcentaje del total de habitantes al $21 \%$, resultado tanto del descenso de la inmigración como del importante volumen de población existente en la actualidad en la provincia de Alicante.

De todo lo cual, se puede deducir que el incremento de la tasa de analfabetismo entre 1960 y 1970 en el grupo de 35/44 años, es producto de la llegada de numerosos inmigrantes con esa edad a Alicante, que, originarios de otras provincias, generalmente meridionales, buscarán en ésta trabajo, en una primera fase, en el sector secundario, y más tarde, en la construcción, ante el crecimiento de la demanda de nuevas viviendas que se produjo en aquéllos años ${ }^{6}$. La posterior caída de sus tasas de analfabetismo está ligada a las campañas de alfabetización que, durante los años setenta, organizaron las autoridades educativas, ante una legislación laboral que llegó a exigir, para según qué tipo de trabajo, la posesión del Graduado Escolar -esto es, haber terminado la segunda etapa de EGB o poseer el título de bachiller elemental-; del Certificado de Estudios Primarios -equivalente a la primera etapa de EGB-; o, al menos, del Certificado de Escolaridad -o de sólo haber estado escolarizado el tiempo mínimo que marcaba la Ley-. La preparación para obtener los dos primeros certificados, se convirtió en la espina dorsal de las campañas de alfabetización antes citadas, llegándose a primar a los maestros que se dedicaron a esta labor socioeducativa. Sin embargo, debemos insistir que ha sido sobre todo la escolarización obligatoria de los jóvenes la que, a la larga, ha hecho disminuir el número de analfabetos en nuestra provincia.

\section{Evolución de los niveles de enseñanza e instrucción}

\subsection{Introducción}

Las fuentes consultadas en este capítulo ${ }^{7}$ no sólo se caracterizan por la escasa o nula

\footnotetext{
${ }^{6}$ Este incremento en la demanda de nuevas edificaciones es resultado, tanto de las nuevas necesidades que generaron dichos inmigrantes, como de la demanda de primeras o segundas viviendas por parte de turistas nacionales o extranjeros.
}

\footnotetext{
${ }^{7}$ Se han consultado, entre otros, los Censos de población de los años comprendidos entre 1900 y 1981 , el Padrón Municipal de habitantes de 1986 y la publicación del INE, Estadística de la Enseñanza, Curso 1980/81.
} 


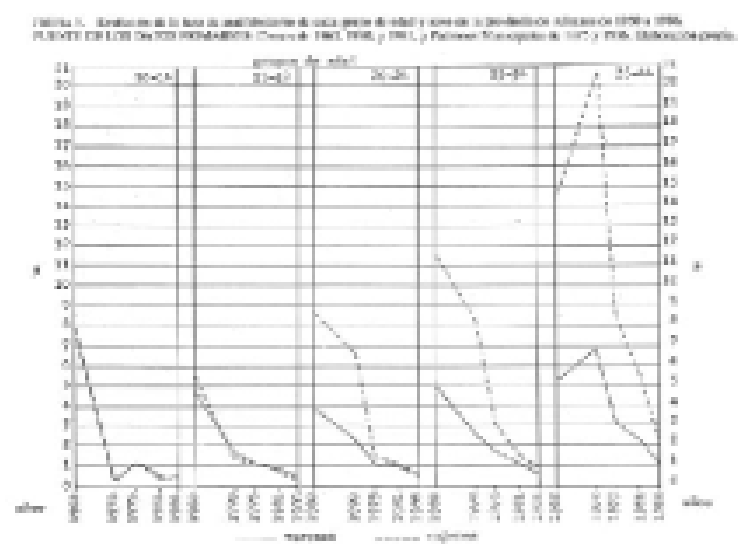

FIGURA 5. Evolución de la tasa de analfabetismo de cada grupo de edad y sexo en la provincia de Alicante de 1960 a 1986.

FUENTE DE LOS DATOS PRIMARIOS: Censos de 1960, 1970, y 1981, y Padrones Municipales de 1975 y 1986. Elaboración propia. 
uniformidad en el modo de agrupar los datos que aparecen en cada una de ellas ${ }^{8}$, sino también por las deficiencias en la formulación de los Censos y el Padrón manejados, las cuales se hubieran podido fácilmente evitar. Todo ello nos ha obligado a establecer clasificaciones distintas según el período analizado, las cuales serán señaladas puntualmente en cada caso, así como a no poder realizar un estudio comparativo de todas las etapas en determinados niveles de estudios, por ejemplo las enseñanzas media y superior.

\subsection{La enseñanza 1900 a 1940}

\subsubsection{Efectivos globales}

El número de estudiantes subió de 34.941 a 119.534 a lo largo de este período, aumento que se hace más significativo si se tiene en cuenta que su porcentaje sobre el total de población pasa del 7,43\% al 19,67\% entre 1900 y 1940 (ver figura 6). Esta positiva evolución resalta aún más si la comparamos con la que experimenta el resto del País Valenciano y el total del Estado Español ya que los incrementos, cuando los hay, son inferiores porcentualmente a los de Alicante. Así, Castellón sube de un 11,16\% a un 13,43\%, Valencia de un 10,03\% a un 17,05\%, el País Valenciano eleva su índice de un $9,48 \%$ a un $17,26 \%$, y España sube su tasa de un $10,62 \%$ a un $17,75 \%$.

El ritmo de crecimiento del número de estudiantes de la provincia de Alicante es asimismo mucho más elevado que el que experimenta su población total, pues si reducimos a 100 sus respectivos valores numéricos en 1900, observamos que, en 1930, mientras los estudiantes alcanzaban el 218, la población total sólo llegaba al 116; y ya en 1940, los primeros conseguían situarse en el 342, mientras que la segunda sólo se hallaba en el 129. Estos valores se significan aún más al compararlos con los del resto del País Valenciano y de España (ver figura 6). Pese ello, las causas más probables de este desigual crecimiento de la población estudiantil en el País Valenciano entre 1900 y 1940 pueden ser: la llegada de inmigrantes de provincias menos cultas que dan una menor importancia a la formación de sus hijos; una excesiva deflacción de los datos de algunos colectivos, como ocurre en la provincia de Valencia en 1940; o una cifra de partida muy baja para la provincia de Alicante y notablemente alta para la de Castellón, sobre todo, tal como veremos más adelante, en la enseñanza primaria.

\subsubsection{Los tipos de enseñanza}

Los escolares de enseñanza primaria se sitúan siempre muy por encima de los que cursan estudios medios/superiores, al representar los primeros al menos el $94 \%$ del total de estudiantes, llegando a suponer, al final del período, el 18,59\% del total de la población. Esta situación, tal vez menos desproporcionada, se repite en el resto del País Valenciano y en España. (ver figura 6). Por otro lado, los efectivos de cada uno de estos dos tipos de enseñanza se han visto sensiblemente incrementados en la provincia de Alicante a lo largo del período, contabilizándose una subida porcentual ligeramente mayor en los estudios primarios que en los medios/superiores, lo que no ocurre en los otros colectivos analizados

\footnotetext{
${ }^{8}$ Este hecho es justificable si se tiene en cuenta: por un lado, el espacio de tiempo tan dilatado que abarca, y por otro, los importantes cambios que en todos los órdenes se han dado en la vida de este país.
} 


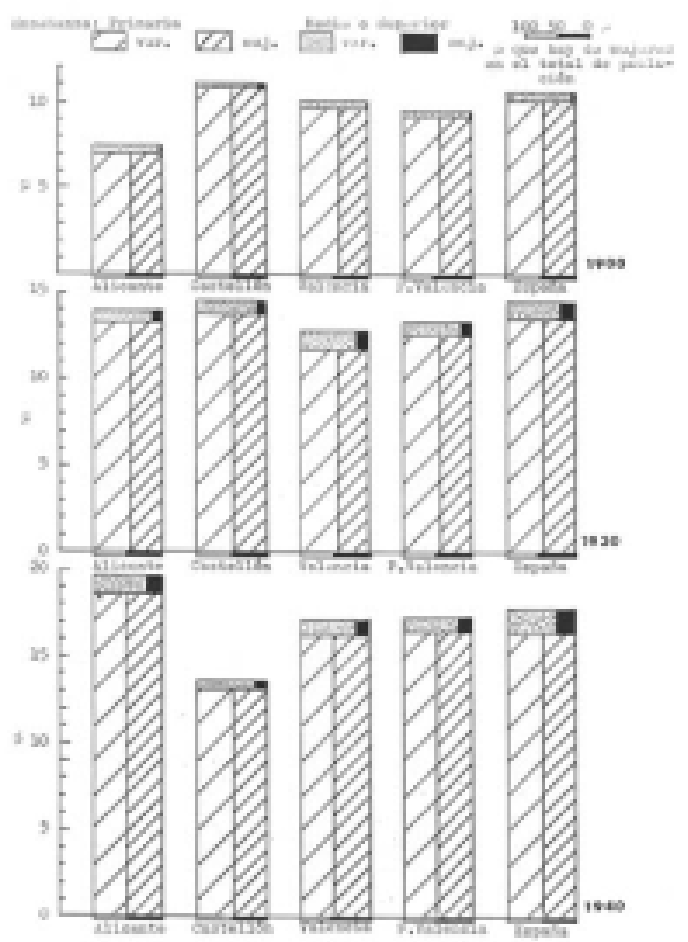

FIGURA 6. Los estudiantes de las provincias de Alicante, Castellón y Valencia, del País Valenciano y de España, en \% del total de población, según el tipo de enseñanza y el sexo, en los años 1900, 1930 y 1940. 
en donde el crecimiento de estos últimos puede llegar a multiplicar por siete el que se da en la enseñanza elemental, como ocurre en la provincia de Valencia entre 1900 y 1930.

Esta diferente manera de evolucionar el estudiantado en Alicante respecto al resto del País Valenciano y el Estado español, bien pudiera deberse a la escasa o nula existencia de centros de estudios medios y, sobre todo, superiores en dicha provincia durante aquellos años. Todo lo cual, acentuará el hecho, por otro lado general, de que, por lo menos hasta 1950, a todo aumento en el porcentaje de alumnos de enseñanza elemental sobre el total de población, le suela seguir una disminución en la tasa de los que cursan estudios medios o superiores; ello es debido a que cuando es escaso el porcentaje de alumnos en el total de población, éstos suelen pertenecer a capas socioculturales elevadas y por tanto con más posibilidades de seguir estudios fuera de su lugar de residencia; por contra, al masificarse la enseñanza primaria, los estratos sociales a los que pertenecen los alumnos también se diversifican, siendo por ello más difícil que se den porcentajes altos de estudiantes que, al terminar dicha enseñanza, puedan ampliar sus estudios, sobre todo si para ello necesitan desplazarse fuera de su provincia.

\subsubsection{La enseñanza según el sexo}

Los varones son más numerosos que las mujeres en el estudiantado de la provincia de Alicante durante todo el período: sólo en 1940 la cifra absoluta de alumnas empezará a ser superior a la de alumnos, aunque manteniendo un peso específico menor que el que tienen estos últimos -en dicho año, aquéllas sólo representaban el 50,21\% del total de los estudiantes, mientras que el 52,25\% de la población eran mujeres-. Este mayor aumento del alumnado femenino respecto del masculino -entre 1930 y 1940 llega a duplicar aquél el crecimiento de éste- es debido, en gran medida, al notable incremento del número de alumnas en la enseñanza primaria y del espectacular avance porcentual del alumnado femenino en las enseñanzas media y superior. Al respecto, no conviene olvidar la labor realizada durante la segunda República por parte de las autoridades para elevar la condición sociocultural de todos los ciudadanos en general y de las mujeres en particular'.

Una situación muy semejante es las que se da en la provincia de Valencia y en el País Valenciano, aunque en ambos casos sólo será en la enseñanza primaria en donde superará, si bien por poco, el número de alumnas al de alumnos. Por contra, en la provincia de Castellón y en España, pese a que concluyen el período con un aumento porcentual mayor en el alumnado femenino que en el masculino, las mujeres siguen presentado cifras absolutas más bajas que los varones.

Este incremento del estudiantado, en general, y de la enseñanza primaria y de las alumnas, en particular, coincide con una importante caída de las tasas de analfabetismo, sobre todo entre la población más joven; todo lo cual viene a confirmar la hipótesis, anteriormente enunciada, de que el descenso del analfabetismo y la escolarización de los habitantes con menor edad suelen ir unidos.

\footnotetext{
${ }^{9}$ En el libro de Femando MILLÁN, La Revolución laica: de la Institución Libre de Enseñanza a la escuela de la República, editado por Bernardo Torres en Valencia en 1983, puede leerse en la página 241 y siguientes cómo subió el porcentaje que se dedicaba a Instrucción Pública en los Presupuestos del Estado de la época de la Monarquía a la de la República, al pasar de un 4,3\% del total de los mismos en 1902 a un 6,5\% en el año 1933, y ello a pesar de encontrarse inmersa la economía española en la Crisis del 29. Igualmente, en la página 221 y siguientes de dicha publicación pueden encontrarse textos legales de la Segunda República sobre la instrucción de la mujer y la coeducación en las escuelas.
} 


\subsection{La enseñanza y la instrucción 1950 y 1960}

\subsection{Introducción}

Los niveles de enseñanza/instrucción que aparecen en los censos de 1950 y 1960 son: la Enseñanza Primaria, superada la cual se obtenía el Certificado de Estudios Primarios (a partir de ahora CEP); las Enseñanzas Profesionales, que cursadas tanto en centros públicos como privados, capacitaban para el ejercicio de alguna profesión que no requería título oficial (mecanógrafos, taquígrafos, mecánicos, etc.) excluyendo los trabajos en régimen de aprendizaje; las Enseñanzas Medias, en ellas o bien se recibía una preparación básica para futuros estudios medios o superiores, o bien se obtenía una titulación oficial que daba acceso a ciertos puestos de trabajo de grado medio, técnicos o profesionales (peritos, ayudantes de ingenieros, maestros de primera enseñanza, aparejadores, etc.); y las Enseñanzas Superiores, que cursadas en facultades o escuelas superiores universitarias, permitía obtener el título de licenciado o doctor.

\subsubsection{La enseñanza}

\subsubsection{Efectivos globales}

El número de estudiantes de la provincia de Alicante, pese a experimentar un cierto aumento entre 1950 y 1960, al pasar su cifra absoluta de 90.648 a 99.735, sufrió un ligero descenso porcentual respecto al total de población, al reducirse su porcentaje de un $14,30 \%$ a un 14,02\%. Esta será asimismo la evolución que seguirán los otros colectivos de referencia; así, lo más común es que frente a pequeños aumentos de las cifras absolutas hallemos también reducidos descensos de sus tasas respecto al total de población (ver figura 7).

Esta evolución está ligada al desarrollo socioeconómico que experimenta durante aquellos años el País Valenciano y, sobre todo, la provincia de Valencia; el cual atraerá a nuestra Comunidad a numerosos inmigrantes, convirtiendo a Valencia, que era la provincia con mejor tono económico, en centro de destino de buena parte de los efectivos recién llegados. Ahora bien, si tenemos en cuenta que el inmigrante de esta primera oleada se caracteriza, fundamentalmente, en que es varón, joven, en edad laboral y con un nivel instructivo bajo, no nos puede extrañar el importante deterioro que sufren los niveles de enseñanza masculinos a lo largo del período, sobre todo en las zonas que recibieron más población foránea, en donde incluso, como es el caso de Valencia, las mujeres verán reducir sus efectivos totales ${ }^{10}$.

\subsubsection{Los tipos de enseñanza}

La enseñanza primaria es con mucho, tal como ocurría en la etapa anterior, la que suma la mayoría de estudiantes, acaparando en 1950 el 92\% del total del alumnado y en 1960 el $83 \%$; igualmente y en esos mismos años, representaba el 13,21\% y el 11,67\% del total de la población, respectivamente. A este tipo de enseñanza le sigue la media que, con un $5 \%$ y un 14\% de los estudiantes en los antedichos años sólo empezará a ser significativa en 1960. En último lugar se sitúan las enseñanzas profesional y superior, las cuales apenas

\footnotetext{
${ }^{10}$ Ver los trabajos de TEIXIDOR DE OTTO, M. ${ }^{\text {a J } .: ~ « D e m o g r a f i ́ a ~ d e ~ l o s ~ i n m i g r a d o s », ~ C u a d e r n o s ~ d e ~ G e o g r a f i ́ a, ~}$ núm. 20, Valencia, 1977, pp. 11-20; «El trabajo de la mujer inmigrada», Cuadernos de Geografía, núm. 28, Valencia, 1981, pp. 105-117; « «Población activa de la ciudad de Valencia», Cuadernos de Geografía, núm. 15 , Valencia, 1974, pp. 25-46.
} 


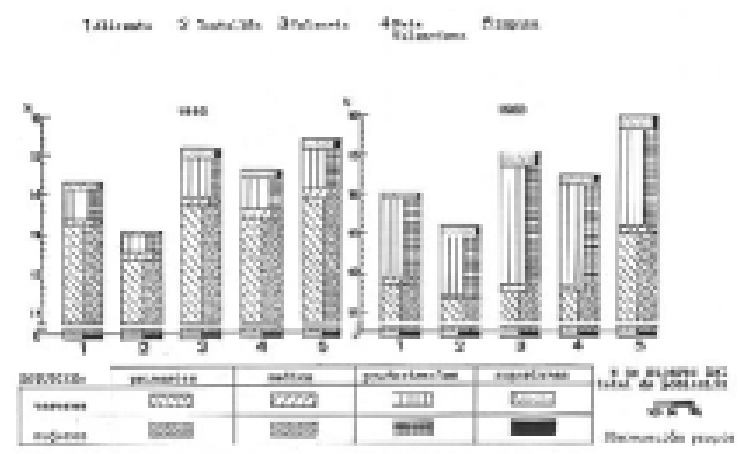

FIGURA 7. Los estudiantes de las provincias de Alicante, Castellón y Valencia, del País Valenciano y de España, en \% del total de población, según el tipo de enseñanza y el sexo, en los años 1950 y 1960. 
alcanzarán el $1 \%$ del total de alumnos al final del período. Por otro lado, cada tipo de enseñanza presenta una evolución distinta; así, mientras que la primaria ve reducir sus efectivos e índices entre 1950 y 1960, tal como ocurre en la superior -aunque con una caída porcentual mucho mayor-, la enseñanza profesional y, sobre todo, la media elevan tanto sus cifras absolutas como sus porcentajes (ver figura 7). Este no es el desarrollo que sigue el resto del País Valenciano, pues en él, la disminución de los efectivos e índices afecta a la enseñanza primaria y a la profesional, mientras que, al contrario de lo que ocurre en Alicante, la superior se une a la enseñanza media a la hora de incrementar sus cifras absolutas y porcentuales.

La anteriormente citada disminución de la tasa de estudiantes de enseñanza primaria, que no llega a ser compensada en modo alguno por los incrementos que se producen sobre todo en la media, es fiel reflejo del estancamiento que experimentan las tasas de natalidad en las tres provincias del País Valenciano de 1940 a 1957, momento en que, salvo en l'Horta de Valencia, se inicia una clara recuperación en toda la comunidad autónoma ${ }^{11}$.

\subsubsection{La enseñanza según el sexo}

El estudiantado femenino de la provincia de Alicante es, en los dos años considerados, menor que el masculino, tanto en cifras absolutas como porcentuales. Por otro lado, si bien la situación que encontramos en el resto del País Valenciano y en el conjunto del Estado Español es muy parecida, cabe destacar que la mujer tiene en ambos colectivos de referencia un peso específico mayor que en Alicante, sobre todo en aquella enseñanza con menor implantación en dicha provincia, tal y como ocurre con la superior (ver figura 7).

Esta situación traerá como consecuencia aumentos más jugosos en el peso específico femenino que en el masculino durante el período, sobre todo en las enseñanzas media y profesional -en esta última, el porcentaje que hay de ellas en el total de alumnos alcanza en 1960 el 47,24\%, lo que las va a situar casi al mismo nivel al que llegaron en los estudios primarios en ese mismo año, un 48,17\% -. Tal vez, la única excepción sea la enseñanza superior, en donde el importante descenso que se produce en sus efectivos, reducirá su implantación de un 11,08\% a un 7,65\%. La evolución de los otros colectivos de referencia es bastante similar a la que acabamos de exponer.

\subsubsection{La instrucción}

\subsubsection{Efectivos globales}

La población con estudios terminados experimentó un notable crecimiento en la provincia de Alicante entre 1950 y 1960, al pasar de 387.240 a 457.027 el número de sus efectivos, subiendo el porcentaje que representaban en el total de los habitantes de un $61,07 \%$ a un $64,24 \%$. Este aumento, que será superior incluso al experimentado por la propia capital, se hace más evidente al reducir a 100 las cifras de 1950, pues, mientras que la población con algún estudio terminado alcanza el índice 118 en 1960, los habitantes de la provincia se quedan en el 112. Los otros colectivos de referencia presentan asimismo avances muy importantes, destacando el que se da en la provincia de Castellón, que eleva su tasa del 61,06\% al 65,56\% en el mismo período (ver figura 8).

\footnotetext{
${ }^{11}$ Ver el artículo de SORRIBES Josep, «Demografía, urbanización y crecimiento económico en el País Valenciano (1800-1980)», publicado en Estructura Social al País Valencià, Diputació de Valencià, Valencia, 1982, p. 182 y siguientes.
} 


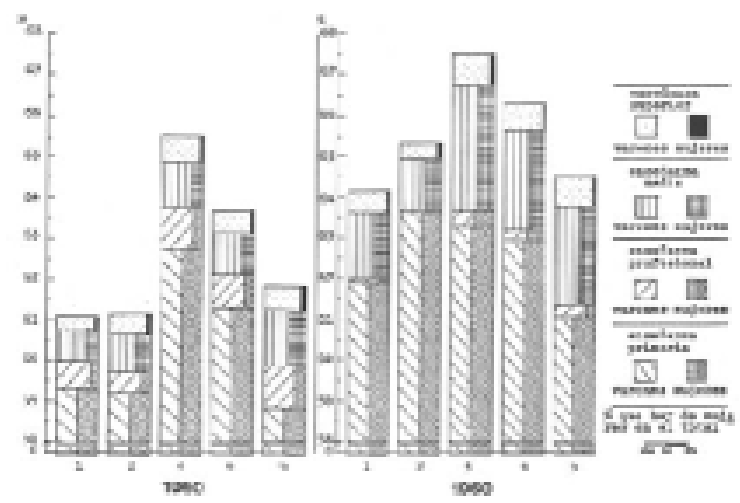

FIGURA 8. Estructura de la población con estudios de las provincias de Alicante, Castellón y Valencia, del País Valenciano y de España, en \% del total de habitantes, según el nivel de instrucción y el sexo, en los años 1950 y 1960. 1. Alicante; 2. Castellón; 3. Valencia; 4. País Valenciano; 5. España. Elaboración propia. 


\subsubsection{Los tipos de instrucción}

La población con sólo estudios primarios es, con mucho, la que suma la mayoría de instruidos en los dos censos consultados, ya que representan en 1950 y 1960 respectivamente, el 59,34\% y $61,89 \%$ del total de la población y el $97 \%$ y el $96 \%$ del total de los habitantes con estudios terminados. Por otra parte, en los otros tipos de instrucción encontramos una evolución muy desigual entre ambos años; así, al mismo tiempo que la población con estudios medios incrementa su tasa notablemente, la que los tiene superiores, sólo experimenta un modesto crecimiento, mientras que los que poseen una instrucción profesional, reducen significativamente su porcentaje -ello está relacionado con el trasvase de efectivos que se produce entre este último tipo de enseñanza y el bachillerato- (ver figura 8).

En cuanto a los otros colectivos de referencia, podemos señalar que la provincia de Valencia y el conjunto de la Comunidad Autónoma presentan, en ambos años, una situación mejor que Alicante, pues además de obtener porcentajes más altos en cada tipo de instrucción, hallamos que éstos son cualitativamente mejores, ya que sus tasas de población con estudios medios y superiores son muy elevadas para la época. Asimismo la provincia de Castellón y la media del Estado Español presentan una situación mejor, si exceptuamos el ligero retroceso que se da en la instrucción superior en la provincia de La Plana (ver figura 8).

\subsubsection{La instrucción según el sexo}

El porcentaje de población femenina con estudios terminados respecto del total de mujeres de la provincia de Alicante es, en los dos censos, menor que la tasa de varones con algún tipo de instrucción; de tal modo que el porcentaje de la población masculina supera al de la femenina: en 1950, en 7,02 puntos porcentuales, y en 1960, en 6,82 puntos. Por otro lado, aunque el peso específico que tiene la mujer en la población instruida disminuye en 0,32 puntos a lo largo del período, como quiera que también disminuye el porcentaje de mujeres en el total de habitantes de la provincia -en 0,54 puntos-, la población femenina no verá empeorada su situación (ver figura 8).

Este panorama se repite cuando se analizan cada uno de los tipos de instrucción; así, al presentar ambos sexos un incremento porcentual tan parecido en los estudios primarios, medios y superiores, y una disminución de la tasa tan similar en los profesionales, la desventaja en todos ellos de las mujeres respecto de los varones apenas sufre variación. Por otra parte, tanto en los varones como en las mujeres, son los estudios primarios, lógicamente, los que acaparan el porcentaje más alto de población con algún tipo de enseñanza terminado; la pequeña disminución porcentual que hallamos en este tipo de estudios es tan reducida, que apenas afecta a la importancia que tiene este tipo de instrucción.

Los otros colectivos de referencia presentan una situación similar a la que acabamos de ver, si exceptuamos la provincia de Castellón, en donde se produce al unísono una disminución, en ambos sexos, de la población con estudios superiores; y la de Valencia, en la que apenas se reducen las mujeres con instrucción profesional.

De todo lo cual, podemos deducir que la mejoría que experimenta la instrucción de los habitantes en la provincia de Alicante es debido a que parte de tasas muy reducidas y, por consiguiente, los notables incrementos que se producen en los porcentajes de población con estudios medios y, sobre todo, con instrucción superior, es más bien resultado de una cifra inicial anormalmente baja para el desarrollo socioeconómico de la provincia (ver 


\section{Cuadro III \\ GRADOS DE ESTUDIOS EN 1970 Y 1981}

$\begin{array}{ll}\text { 1. }{ }^{\text {er }} \text { Grado: } & \text { Enseñanza Primaria/ } \\ & \text { Primera Etapa de EGB }\end{array}$

2. ${ }^{\mathrm{o}}$ Grado: $\quad 1^{\mathrm{er}}$ Ciclo:

$2^{\circ}$ Ciclo:

3. ${ }^{\text {er }}$ Grado: $\quad 1^{\text {er }}$ Ciclo:

$2^{\circ}$ Ciclo: antiguo Bachillerato

elemental/Segunda Etapa de EGB

antiguo Bachillerato Superior/BUP/COU

estudios universitarios de Grado Medio/Diplomatura Universitaria

estudios universitarios Superiores/Licenciatura/Doc torado

cuadro II), que sólo es explicable a partir de la escasez o ausencia de centros en donde cursar estos estudios. En el caso de las mujeres, la situación se complica, pues a lo anterior hay que añadir la consideración sociocultural que éstas han tenido en nuestro país hasta fechas recientes, lo que ha representado un obstáculo más a la hora de desplazarse fuera de su lugar de residencia para continuar estudios.

\subsection{La enseñanza y la instrucción en 1970 y 1981}

\subsubsection{Introducción}

La instrucción/enseñanza tiene, según los Censos de 1970 y 1981, tres grados (ver Cuadro III). Sin embargo, para hacer menos farragoso nuestro análisis, hemos optado por renunciar a atender todas las clasificaciones, con toda la riqueza de matices que aparecen en ambos Censos de población, agrupando los datos de acuerdo a los mismos criterios mantenidos hasta ahora.

Finalmente señalamos que no coincide la población que se considera en cada censo; así: respecto a la enseñanza, mientras que en el de 1970 se atiende a toda la población que cursaba estudios, en el de 1981 sólo se considera a la que tiene 4 años o más; y en cuanto a la instrucción, mientras que el de 1970 tiene en cuenta al total de habitantes, el de 1981 sólo da cifras de los que tienen 10 años o más. Sin embargo, no creemos que la diferencia en conjunto sea importante, pues es difícil hallar tantos estudiantes con menos de 4 años, como niños con menos de 10 años con algún título.

\subsubsection{La enseñanza}

\subsubsection{Efectivos globales}

El número de estudiantes existentes en la provincia de Alicante aumentó considerablemente entre 1970 y 1981 al pasar sus efectivos de 194.076 a 268.045; asimismo se elevó su porcentaje respecto del total de población de un $21,05 \%$ a un 23,32\%. Una evolución similar hallamos en el resto del País Valenciano y del Estado Español (ver figura 9). 


\subsubsection{Los tipos de enseñanza}

La enseñanza de primer grado -agrupa a la educación preescolar con los estudios que se desarrollan en la primera etapa de EGB- presenta, en los dos censos consultados y tal como ocurría en años anteriores, las tasas más altas de alumnos, pues representan en 1970 y en 1981, el $15,82 \%$ y el $13,17 \%$ del total de población de la provincia, respectivamente. Sin embargo y tal como puede comprobarse, sus índices no son tan elevados como entonces, antes bien, parece que incluso se agudiza la tendencia a la baja detectada en el estudio que realizamos de aquellos períodos, así, su tasa del total de alumnos pasa entre dichos años de un 75\% a un 57\%; igualmente, este tipo de estudios es el que menos crece a lo largo del período, al aumentar sólo cuatro puntos porcentuales en el índice 100 de 1970 a 1981, un incremento, que tal como veremos, es a todas luces muy inferior al que experimentan los otros niveles de enseñanza (ver figura 9).

A los estudios de primer grado le sigue en importancia la enseñanza en la segunda etapa de EGB, cuya tasa respecto del total de población crece entre ambos años de un 3,63\% a un 5,39\%, pasando su cuota del total de estudiantes del 17\% al 23\% entre 1970 y 1981, al tiempo que incrementa su índice 100 en 84 puntos porcentuales. Por otro lado y a pesar de ocupar el tercer lugar en cifras absolutas y porcentuales, son los alumnos de BUP/COU y FP los que más incrementan sus índices en este período, puesto que si bien su tasa del total de población sólo asciende de un $0,96 \%$ a un 3,52\% entre estos años, su porcentaje del total de estudiantes pasa de un 5\% a un 15\% a lo largo del período, y reduciendo a 100 las cifras de 1970, en 1981 alcanza el índice 455. Asimismo, también crecen los efectivos en la enseñanza superior, siendo el aumento más importante el que se da en la de segundo ciclo. En las enseñanzas universitarias del primer ciclo, las subidas absolutas y porcentuales son más discretas que las anteriores, si exceptuamos a las producidas en los alumnos de primer grado.

En conjunto, los otros colectivos de referencia, pese de seguir una evolución similar a la que experimenta la provincia de Alicante, presentan un panorama ligeramente mejor, dado que, a la peor tasa de estudiantes de enseñanzas universitarias en la misma, se le une la obtención de unos índices en 1970 también más bajos de alumnos de BUP/ COU y FP, y, con la excepción de Castellón, de segunda etapa de EGB; en 1981, sólo se coloca por encima de estos colectivos en el porcentaje de estudiantes del primer grado del segundo ciclo, y respecto a la provincia de Castellón, en la tasa de alumnos de enseñanzas medias. Todo lo cual justifica de alguna manera el que haya crecido más deprisa el alumnado en Alicante que en estos colectivos, al tener unas cifras de partida netamente inferiores a las de éstos.

\subsubsection{La enseñanza según el sexo}

Las mujeres que, tal como ocurría en el periodo precedente, siguen presentando cifras absolutas y porcentuales de estudiantes más bajas que los varones tanto en 1970 como en 1981, experimentan un crecimiento ligeramente mayor que éstos entre ambos años. Esta situación es bastante similar a la que hallamos en el resto del País Valenciano y en el Estado Español.

\subsubsection{La instrucción}

\subsubsection{Efectivos globales}

La población con estudios terminados en la provincia de Alicante, pese a experimentar un notable crecimiento de su cifra absoluta entre 1970 y 1981-pasa el número de sus 


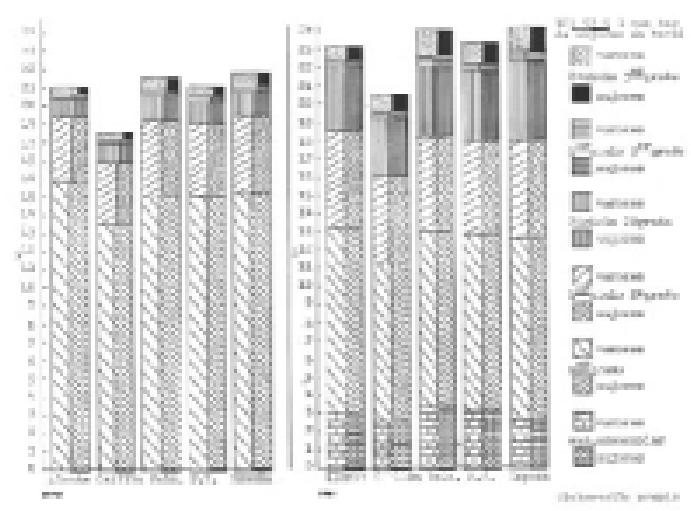

FIGURA 9. Los estudiantes de las provincias de Alicante, Castellón y Valencia, del País Valenciano y de España, en \% del total de población, según el tipo de enseñanza y el sexo, en los años 1970 y 1981. 
efectivos de 440.923 a 553.763-, verá disminuir su porcentaje del total de habitantes de un $60,57 \%$ a un $58,90 \%$. Este descenso en la tasa se manifiesta aún más claramente al reducir a 100 las cifras de 1970 y comprobar cómo en 1981, frente al índice 129 al que llega el total de población, encontramos el valor 125 para los habitantes con algún título (ver figura 10).

Sin embargo, estos índices, que son similares a los que se dan en los otros colectivos de referencia, no reflejan fielmente la realidad, puesto que son resultado de los cambios que se introducen en el Censo de 1981 respecto al de 1970; así, a la hora de contabilizar a los habitantes según sus estudios, mientras que en el de 1970 se considera a la población que no cursa enseñanza alguna, cualquiera que sea su edad, en el de 1981 se tiene en cuenta a todos los habitantes con 10 o más años, se hallen o no cursando estudios, lo cual nos hace suponer, que en este último colectivo deben figurar incluidas muchas personas que todavía están realizando su enseñanza más elemental; lo que queda confirmado al observar el descenso que se produce en 1981 de población con sólo el primer grado de enseñanza, y que es resultado, además de otras causas que más adelante se analizarán, de ésta, puesto que en ese año el grupo de edad en el que teóricamente se cursa la primera etapa de EGB, todavía no se había visto afectado por el descenso de la natalidad que ya empieza a constatarse en la provincia.

\subsubsection{Los tipos de instrucción}

Al descenso que se produce, entre 1970 y 1981, de población con sólo la primera etapa de EGB o el CEP se contrapone un importante crecimiento en los demás tipos de instrucción. Así tenemos que: los habitantes con Graduado Escolar (GE) o bachillerato elemental (BE), incrementan su tasa respecto del total de población de un 1,96\% a un 17,65\% entre 1970 y 1981 ; asimismo, el colectivo de personas con BUP/COU o similar, también elevan su porcentaje de un $1,51 \%$ a un $3,56 \%$ entre los mismos años; por otro lado, los ciudadanos con diplomatura universitaria, aumentan igualmente su tasa de un $1,36 \%$ a un $2,34 \%$; y por último, los licenciados/doctores, también elevan su porcentaje a lo largo del período desde un $0,79 \%$ a un $1,42 \%$ (ver figura 10). En cuanto al resto del País Valenciano y España, podemos señalar que, salvo en la provincia de Castellón, generalmente presentan porcentajes más altos en 1970 que en 1981; sin embargo y pese a ello, hay niveles de instrucción que se han desarrollado espectacularmente, como es el caso de la población con BUP/COU o FP del conjunto del Estado Español, cuya tasa se ha multiplicado por 5 a lo largo de este período.

De cualquiera de las maneras y reduciendo a 100 las cifras de 1970, es el índice de población con GE de Alicante y, en menor medida, de Castellón los que experimentan un crecimiento mayor, casi explosivo, al alcanzarse en 1981 en ambas provincias los valores 1.160 y 1.081 respectivamente. Asimismo y en conjunto, es en estas dos provincias en donde se produce un incremento mayor en el índice 100, de 1970 a 1981, en los diferentes tipos de enseñanza, como resultado de haber partido de una situación peor en cuanto a la instrucción de sus habitantes. En general, las tres provincias del País Valenciano verán incrementados sus efectivos en los niveles de instrucción no elemental, hasta tal punto que, al final del período, los porcentajes en cada uno de ellos se acercarán notablemente a los que encontramos en la media de España, que a su vez es con mucho, la que parte de una situación mejor. 


\subsubsection{La instrucción según el sexo}

El porcentaje de población femenina con estudios terminados respecto del total de mujeres de la provincia de Alicante es, en todo momento, algo menor que la tasa de varones con algún título. Por otro lado y a pesar de que disminuye medio punto, entre estos años, el porcentaje que hay de población femenina en el total de habitantes, las mujeres aumentarán su peso específico en el conjunto de población instruida en 0,68 puntos porcentuales (ver figura 10).

Este panorama es similar al que nos ofrece la situación de cada sexo en cada nivel de instrucción, pues, o bien los incrementos de las tasas, cuando éstos se producen, son mayores en las mujeres que en los varones, o bien los descensos en los porcentajes inciden menos en la población femenina que en la masculina. Por otro lado, es necesario destacar el hecho de que nos encontrarmos ya con un nivel de instrucción en donde las mujeres son predominantes, tanto en cifras absolutas como porcentuales, así, con diplomatura o similar hallamos en 1981, no sólo 925 personas más del sexo femenino que del masculino, sino que su porcentaje es 0,06 puntos más alto, al tiempo que su peso específico es de $52,10 \%$ frente al $51,41 \%$ que hay de mujeres en el total de población.

Si consideramos seguidamente en cada nivel de instrucción el porcentaje que hay de varones y de mujeres respecto del total de población con estudios de cada sexo, hallaremos las siguientes tasas: en 1970, con CEP, el $89 \%$ y el $93 \%$ respectivamente, con BE, el $4 \%$ y el 3\%, con el bachillerato superior (BS), el $3 \%$ y el $2 \%$, con carreras de grado medio, el $2 \%$ en los ambos sexos, y con título superior, el $2 \%$ y menos del 1\%; y en 1981, con la primera etapa de EGB o el CEP, el $56 \%$ y el $60 \%$ respectivamente, con la segunda etapa de EGB o el BE, el $30 \%$ en ambos sexos, con el BUP/COU o BS, el $7 \%$ y el $5 \%$, con diplomatura universitaria o similar, el $4 \%$ en ambos casos, y por último, con título de licenciado o doctor, el $3 \%$ y el $1 \%$. Ante estas cifras, no es muy difícil llegar a la conclusión de que entre 1970 y 1981 se produce en ambos sexos, y sobre todo en la población femenina, un importante cambio cualitativo en su instrucción (ver figura 10).

En los otros colectivos de referencia, el peso específico que tiene la mujer en el conjunto de los instruidos también aumenta relativamente, con la excepción de la media de España, en donde éste se desfasa respecto de la tasa de población femenina en más de medio punto porcentual. Asimismo, en cada uno de los niveles de instrucción, las mujeres van a ir ocupando un espacio mayor a pesar de los importantes incrementos que se producen en las tasas de los varones, sólo en la provincia de Castellón y en la media del conjunto del Estado Español, encontramos un nivel de estudios alcanzados -el primer grado- en donde la población femenina pierde peso específico.

De lo que podemos deducir que la población de Alicante presenta una instrucción de menos calidad que la que ofrecen tanto Valencia como la media de España, al obtener, a partir de la titulación de BUP/ COU en adelante, unas tasas muy inferiores a las de estos colectivos. La principal causa de este peor paisaje instructivo, es la continua llegada a nuestra provincia de inmigrantes procedentes de zonas menos desarrolladas de España, lo que generará un descenso en el nivel medio de instrucción de los habitantes de Alicante.

\section{Conclusiones}

El análisis de los Censos y Padrones de población consultados, nos ha descubierto numerosos errores en la confección, cumplimentación y elaboración de los datos que aparecen en ellos, a los que han sido ajenos, muchas veces, los funcionarios del Instituto 
Nacional de Estadística (INE) o del Ayuntamiento de Alicante, pues nos consta que su encomiable labor se vio turbada en algunas ocasiones por la presión que, políticos sin escrúpulos, ejercieron para, de esa manera, desfigurar la realidad en su propio

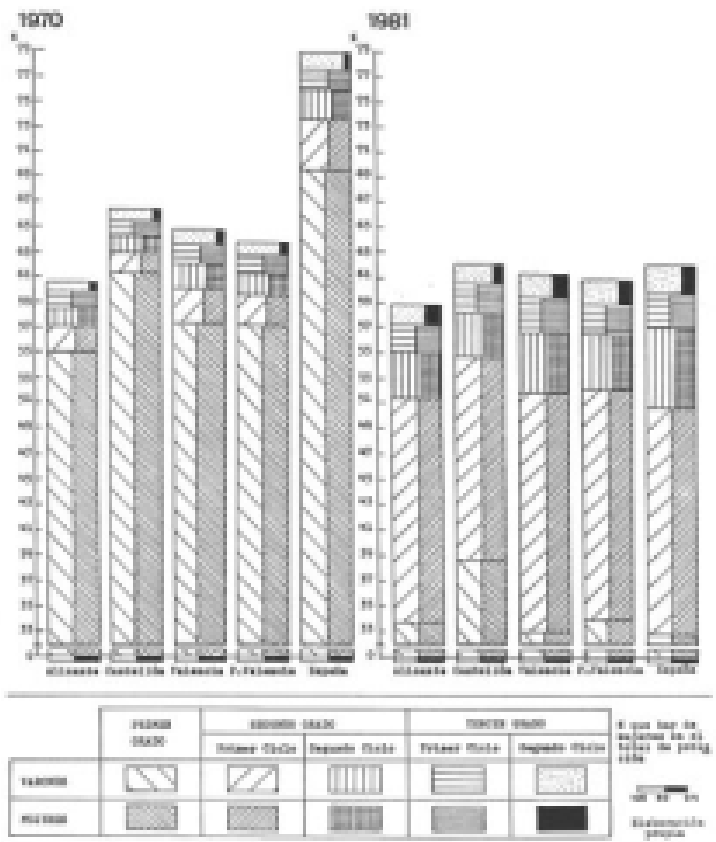

FIGURA 10. Estructura de la población con estudios de las provincias de Alicante, Castellón y Valencia, del País Valenciano y de España, en \% del total de habitantes, según el nivel de instrucción y el sexo, en los años 1970 y 1981. 


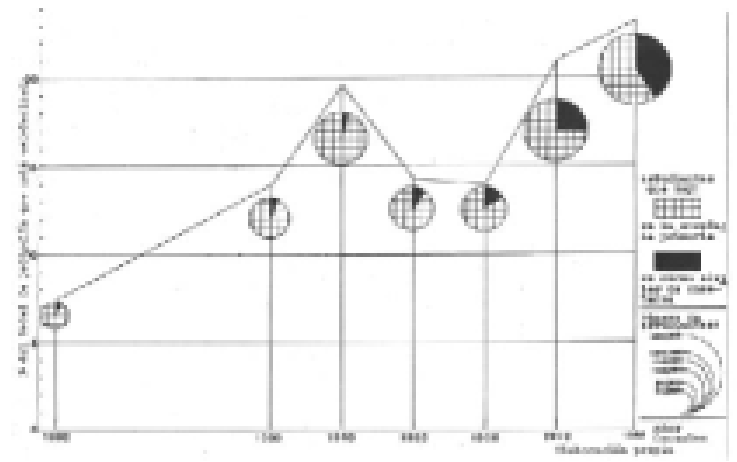

FIGURA 11. Evolución de la población que cursa estudios en la provincia de Alicante de 1900 a 1981, en \% del total de población, en cifras absolutas y según el tipo de enseñanza. 
provecho ${ }^{12}$. Por ello, creemos que es conveniente realizar una depuración de dichos Censos y Padrones.

Por otro lado, en la evolución que experimenta la instrucción y la enseñanza del conjunto de los habitantes de la provincia, se pueden distinguir las siguientes tres etapas (ver figuras $11 \mathrm{y}$ anteriores):

Primera etapa o preinstructiva, que concluye en la provincia de Alicante en 1940 y se caracteriza por la existencia de un alto porcentaje de analfabetos -no desciende por debajo del $30 \%$ del total de población con 10 años o más hasta pasada dicha fecha-, destacando, por lo elevado de sus índices, los jóvenes menores de 20 años, los adultos mayores de 46 y la población femenina; asimismo, entre los escasos escolarizados que hallamos, predominan los varones y los estudiantes de primaria.

Segunda etapa o de la instrucción elemental, que abarca en la provincia alicantina de 1940 a 1970 y que está definida: por una caída sostenida de las tasas de analfabetismo hasta situarse en torno al $10 \%$ en la última fecha -los varones son los primeros en alcanzarla, puesto que parten de una mejor situación, sin embargo, la población mayor de 65 años, sobre todo la femenina, seguirá ofreciendo tasas superiores al $30 \%$ al final del período-; y por un importante crecimiento del número de escolarizados, sobre todo en enseñanza elemental, por un lado, al darse prioridad a la instrucción primaria, y por otro, no aumentar en la misma medida el alumnado de estudios medios y superiores, al faltar centros accesibles a los mismos. Asimismo, durante esta etapa, el peso específico que tienen las mujeres en la enseñanza elemental, sobre todo las más jóvenes, empieza a superar al que tienen los varones, debido a su sex ratio superior.

Tercera etapa o de las reformas educativas, en la que nos hallamos actualmente y que está caracterizada: por una continua reducción del analfabetismo -cuya tasa se sitúa en 1986 por debajo del 5\%-, este descenso será mayor en las mujeres que en los varones, pues aquéllas, en los grupos de edad más jóvenes, obtienen al final de esta etapa unos índices de analfabetismo más bajos que éstos; por alcanzar la escolarización básica en 1986 tasas casi del 100\%, entre los niños de 6 a 13 años, al mismo tiempo que se ampliará dicha escolarización por debajo y por encima de dicha edad -con la educación preescolar y las enseñanzas medias-; y por la sucesión de reformas que experimenta la enseñanza ${ }^{13}$-de ahí el nombre adoptado-, lo que unido a los cambios producidos en la legislación laboral, que impide trabajar a los menores de 16 años ${ }^{14}$, ha traído consigo una mejoría en la escolarización en enseñanzas hasta hace bien poco consideradas no básicas, como la media o superior.

\footnotetext{
${ }^{12}$ Son numerosos los párrafos que encontramos en la Memoria del Censo de 1940 en los que se muestra esta posible desfiguración; así y a modo de ejemplo, podemos señalar aquellos que en el artículo 62, tanto se exige una mayor diligencia en los agentes censales para evitar inscripciones incompletas o exageradas, como se afirma, infantilmente, que el mejor plebiscito de la bondad del recién instaurado régimen son los resultados del Censo, Censo que, por otro lado, difícilmente hubiera podido reflejar en 1940, si no es manipulándolo, los logros que en nuestra comunidad autónoma sólo tuvieron un año para obtenerse.

${ }^{13}$ Bastaría con señalar para ver la importancia de éstas el que desde 1857, en que se aprueba la Ley de instrucción pública de MOYANO, no se había aprobado ninguna Ley general de educación hasta 1970, en que se promulga la Ley General de Educación y financiamiento de la Reforma Educativa.

${ }^{14} \mathrm{Si}$ a lo acabado de señalar sobre legislación laboral se le añade la dificultad de encontrar trabajo, sobre todo por los jóvenes, quedará aclarado el por qué muchos de éstos, antes de engrosar las cifras de paro juvenil, preferirán dedicar unos años más de su vida a su formación, incluso con estudios superiores.
} 